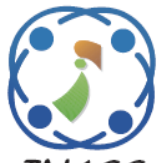

\title{
Schedule-based Optimized Node Recharging Model (SONRM) For Increasing Longevity of Wireless Rechargeable Sensor Network (WRSN)
}

\author{
G. R. Sakthidharan ${ }^{1 *}$ \\ P. Chandra Sekhar Reddy ${ }^{1}$ \\ ${ }^{I}$ Department of Computer Science and Engineering, \\ Gokaraju Rangaraju Institute of Engineering and Technology, Hyderabad, Telangana State, India \\ * Corresponding author's Email: grsdharan@ gmail.com
}

\begin{abstract}
There has been a tremendous growth in research concern in Wireless Sensor Networks (WSNs), due to the broad range of applications in distinctive areas such as environmental observation, security domains, home automation, etc. Despite advantages, the prevalent adoption of WSN, specifically in remote and isolated regions, has become the major challenge, because of this battery constrained network with limited energy source. As advancement, the nodes are framed under Wireless Rechargeable Sensor Network (WRSN) to enhance the lifetime of sensor nodes with the establishment of charging mechanisms. With those considerations, this paper presents a Schedule-based Optimized Node Recharging Model (SONRM), in accordance to the condition of limited capability of mobile charging. In the process of the construction of charging circuit, the SONRM effectively considers the dynamic changes of energy utilization among nodes at different factors and develops a Recharge Scheduling Algorithm (RSA) that helps in finding the next Energy Starving Sensor Node (ESSN) to be recharged. Simultaneously, a model for the establishment of feasible circuit for recharging has also been done for ensuring the effectiveness of charging. During the operation, the node mortality can be reduced by the proposed model with the incorporation of dynamic charging threshold. When compared with the existing mechanisms, the SONRM attains better results on analysing decisive factors such as network longevity, service time, efficient charging, throughput and recharging time.
\end{abstract}

Keywords: Wireless rechargeable sensor network (WRSN), Schedule-based optimized node recharging model (SONRM), Recharge scheduling algorithm (RSA), Energy starving sensor node (ESSN).

\section{Introduction}

In current scenario of WSN research, energy efficiency has become a significant subject to concentrate. The concern about energy efficiency may be endorsed to certain constraints induced by the batteries that are used to power-up those devices. Generally, those batteries are the major power source for WSN devices and featured by limited lifetime, after that they are discarded or charged for further process $[1,2]$. It is apparent that WSN has been the most significant in ubiquitous computing applications like disaster management, rescue operations, military observations, ecological and structural surveillance, health care monitoring, security inducement and wild life tracing, etc [3, 4].
Due to that orientation of such applications, the sensor nodes deployment would be generally in an isolated environment, which is difficult to access and also with battery constraints. Therefore, the lifespan of those sensor nodes has become a major threat. There have been several approaches are developed for increasing the battery lifetime of the sensor nodes, thereby increasing the network longevity of the Sensor Networks [5, 6].

As mentioned, the sensor nodes are distributed over the inaccessible environment for observing the ecological modifications based on dynamic temperature, pressure, motions, vibration, pollutants or some other location based predefined or distinctive activities. Here, the deployed sensor nodes are required to send the gathered data to the 


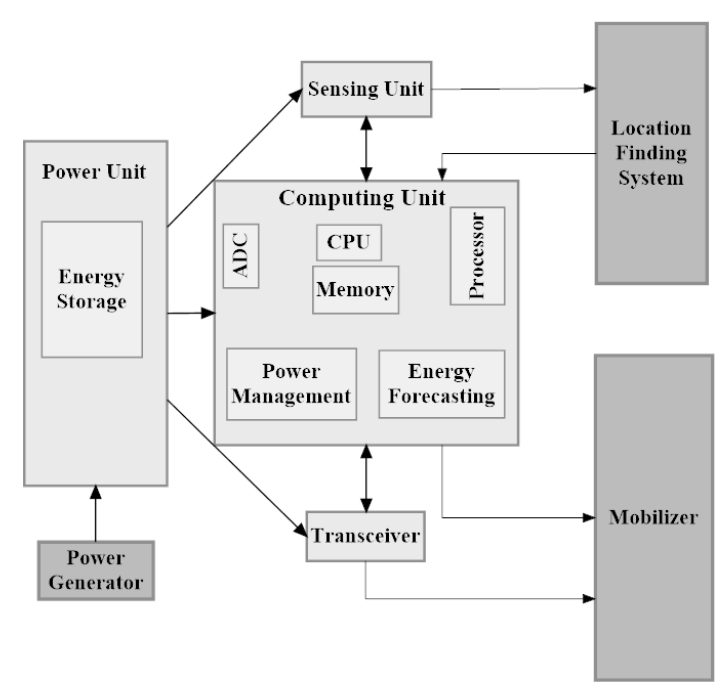

Figure. 1 Main components of sensor node

Base Station (BS) continuously, in standard time periods. Due to the constrained power source in all nodes, there are so many approaches have been proposed for efficient energy management in WSN. Generally, the overall energy source of a sensor node is utilized by the four major units, called, computing unit, power unit, sensing unit and the unit for communication. The Fig. 1 portrays the main components of a sensor node.

The sensing unit presented in the top contains sensors to sense the environment and gathers relevant information. Further, the computing unit comprises Analog-to-Digital Convertor (ADC) in which the gathered analog signals are formatted to digital waves and forwarded. A small storage unit is also presented in the computing unit to process with the sensor node co operations. Then, the communication unit has the transceiver to provide the communication between the sensor nodes to the network. It is apparent that the sensor nodes have constrained power source, the node contains a power unit with some source of energy to consume during operations. Location finding system is an additional component for acquiring better information about the surroundings, in which the node is distributed. And, mobilize is a component for giving the sensor's mobility aspect on the WSN. Among all units, the communication unit involves in data transmission and reception, consumes considerably more amount of available energy [7].

A recent advancement in wireless power transfer technique for resolving the energy constrained problem that can make it possible to recharge nodes for enhancing the network lifespan. It is typically named as Wireless Rechargeable Sensor Networks (WRSN), which is effectively used for recharging sensor nodes, thereby, provides supplement power for the sensor devices to perform. When this charging technique is applied to the portable charger, the charger has to be scheduled for recharging the nodes in the considered network. Further, the mobile chargers involve in recharging the sensor batteries and gather information to pass through the nodes based on the predefined route simultaneously [8]. This may enhance the network lifetime, but it has some practical complications to execute.

Practically, the amount of information acquired from various nodes in distinctive environments is different, which may cause variations in energy consumptions on each sensor node because of frequent changes. Thus, it is essential to frame a scheduling model with efficient considerations of the different energy consumption rates of the deployed nodes. Moreover, the proposed model in this paper considered the varied energy utilization of nodes and the distance of portable charging device, developed a Schedule-based Optimized Node Recharging Model (SONRM). Initially, a computational model for predicting the rate of energy consumption has been determined. With that computational model, Recharge Scheduling Algorithm (RSA) has been developed and that algorithm helps in finding the next Energy Starving Sensor Node (ESSN) to be recharged.

\subsection{Contributions of the paper}

There are three major contributions in this paper, as follows:

- An Efficient Computational model for predicting the node's energy consumption rate has been incorporated, since the deployed nodes have undergone variant task loads due to the dynamic environmental changes. With respect to the model results and the distance between the Portable Charging Device (PCD) and the sensor nodes, Recharge Scheduling Algorithm (RSA) has been incorporates, with adaptive nature of environmental changes.

- The PCD is considered to be accumulated with limited energy and the charging circuit is framed for ensuring the durable operations of WRSN. The model has been further optimized by defining the dynamic charging threshold. This process minimizes the charging delay and enhances the overall network performance.

- Based on the proficient theoretical examinations, the simulated experimentation has been carried out with the Network Simulator NS-3 tool. The results are analyzed and comparative discussions have been made for evidencing the outstanding results of the proposed model with respect to the decisive 
factors such as average service time, network longevity, recharging time and charging efficiency.

The rest of the paper is organized as follows: Section 2 describes a reflection on the study of related works. Section 3 provides the problem statement in short. Section 4 lays out the detailed work process of the Schedule-based Optimized Node Recharging Model (SONRM) for increasing network longevity. The experimental results and comparison charts are given in section 5. Finally, section 6 concludes the paper with some paths for future enhancement.

\section{Related works}

As discussed earlier, the sensor nodes are working with batteries, which are energy limited [911]. For solving the battery constrained issues and energy refilling problems in wireless rechargeable sensor networks, various researches have been accomplished and models are developed. On the whole, the approaches can be categorized into two, as, on-demand recharging and periodical recharging. So many models have been developed based on the periodical recharging methodology, with that, the mobile charger travels along the nodes in WSN periodically and provide energy to the nodes. But, it can be applicable only in scenario in which the consumption of power among nodes is stable. Nevertheless, the energy consumption of sensor nodes varies due to the dynamic environmental nature, which is not appropriate for applying periodical recharging process. On the contrary, the efficient recharging process falls under WRSN ondemand class.

In [12], the mobile charging mechanism for charging the sensor nodes is controlled with the model called Nearest First and Recent Rarest. A game theoretical collaborative charging scheme has been proposed in [13], in which the mobile charger involves in charging the weaker nodes as well as adding power to another mobile charger that is having less energy, but need to recharge many nodes. Further, a greedy algorithm based charging methodology has been developed in [14]. The sensor nodes have been ranked based on the energy requests and the residual energy rates, for providing the charging service to the network. The lifespan of the network is estimated with the first dead node in that particular network.

Moreover, in the works of [15] and [16], the sensor nodes in WRSN have been recharged using the mobile charging vehicle based on the ascending ordered priorities. The process of charging has been accomplished with the help of queue model and receiving requests for energy from the nodes are accumulated in the buffer pool. The method called Nearest Job Next with Pre-emption (NJNP) method was developed for the implementation of the defined on-demand recharge process. In that, the mobile charger intended to select the most nearest node for recharging first, rather considering the residual energy of the sensor nodes. This may cause the sensor nodes that are farer from the mobile charger to dead, thereby reduces the network lifetime.

In [17], the authors have presented an Enabling Temporal and Distantial Priority (TADP) based scheduling scheme for charging sensor nodes. The scheme operated without the consideration of energy consumption variations of distributed sensor nodes due to the dynamic environmental nature. In another way, On-Demand charging process impacts edge computing in greater way [18]. For replenishing the power from the mobile charger for the maximum possible sensor nodes, an efficient charging route is required. In the work [19], the factors such as utmost energy capacity of mobile charger and the residual energy of sensor nodes are considered for estimating, whether the mobile recharger accomplishes the desired tack and back to the BS before depleted its complete energy.

The battery capacity of the mobile charger has been decided based on the pre-fixed network lifetime to attain. The estimation of the adaptive mobility speed and recharging rate of the mobile charger has also been derived based on the network life span [20]. In [21], for increasing the charging efficacy, the utmost moving distance of the charging vehicle was considered. In addition, for recharging the nodes, the sensor nodes in the network are clustered in accordance to their charging sequence and geographical positions are given for consideration. But, the motive is to reduce the charging sequence by computing the appropriate location and optimal charging rate of the mobile charger [22]. Further, in [23], single mobile charger scheme based Hybrid Clustering Charging Algorithm (HCCA) has been proposed. The algorithm was used to estimate solid energy level for reducing the travelling time of mobile chargers and the charging duration of sensor nodes.

\section{Problem statement}

In realistic network environments, the energy utilization of each sensor node differs based on time due to the environmental influences and locations changes. In this paper, Schedule-based Optimized Node Recharging Model (SONRM) that works on 
on-demand charging process has been proposed majorly resolves the following three issues:

1. The Portable Charging Device (PCD) serves as mobile charger here, is assumed to be charging single node at an instant. When the PCD gets several requests, then the requests are to be efficiently scheduled for processing to avoid the sensor nodes from inactivation because of energy depletion.

2. For reducing the long waiting time of other nodes to get charging, the model has been developed in such a way to refill required amount of energy for the nodes to be in activation instead of recharging completely to their battery level. This can be performed by the inducement of dynamic charging threshold rate.

3. Since the PCD has limited energy source, the average service time will be higher and number of recharged nodes are also limited, hence an appropriate charging circuit is needed to make the nodes to be refilled, when the energy source is found to be low.

Hence, by solving these described issues, the network lifetime can be prolonged and the average survival rate of nodes can also be maximized.

\section{Work process of schedule-based optimized node recharging model (SONRM)}

This paper proposed a Schedule-based Optimized Node Recharging Model (SONRM) to recharge the sensor node energy and the possible conditions are formulated to ensure whether the Portable Charging Device (PCD) can provide the prolonged activation of Wireless Rechargeable Sensor Network.

\subsection{System model description}

As given in the Fig. 2, for an assumption, there are ' $\mathrm{N}$ ' number of rechargeable sensor nodes are distributed in the defined rectangular field in random manner. ' \EY』_mx' denotes the maximum battery level of the distributed sensors. Moreover, the nodes are installed with a power harvester to receive the energy from the PCD. The PCD is the wireless energy transferring device that can move at a constant speed ' $v$ ' with the maximum energy transferring capacity as ' 『ET】_mx'. The deployed nodes observe the surrounding objects; collect relevant data and forwards to the base station. When the energy of the sensor nodes falls under the predefined dynamic charging threshold ' 『EY】 thresh', energy request will be forwarded to the PCD from the corresponding sensor node for refilling energy. The request message contains the ID and the energy utilization rate of the sensor node. All the energy requests that are sent by different nodes are preserved in the buffer pool at the receiver side of PCD. Then, using the Recharge Scheduling Algorithm (RSA), the requests from the buffer pool are resolved by one-to-one charging mode, in sequential manner.

\subsection{Schedule-based optimization node recharging model depiction}

This section describes schematic formulation of the Schedule-based Optimized Node Recharging Model (SONRM) for resolving the issues illustrated in the section 3. The model comprises four main parts, computational model for Energy Utilization Rate of Senor nodes, selection of next Energy Starving Sensor Node (ESSN), framing of Recharge scheduling Algorithm (RSA) and adoption of Dynamic Charging Threshold.

\subsubsection{Computational model for energy utilization rate of senor nodes}

The energy utilization rate of sensor node ' $x$ ' at an instant ' $t$ ' is given as ' 『ER】_ $x(t)$ '. As given in the following derivation (1), the computational model of Energy utilization rate is derived in two parts: state_of_charging and state_of_non-charging. From (1), it is to be stated that, ' $\lambda$ ' is the control parameter, ranges from $(0<\lambda<1)$ and the residual energy of node ' $x$ ' at an instant ${ }^{6} t$ ' is given as ' 『RE 】_x (t)'. In a case of non-charging state of the sensor node, it is required to race with other demanding nodes for Portable Charging Device (PCD). Hence, the energy utilization rate of each sensor node has been computed in every ' $\mathrm{T}$ ' period of time to determine the charging sequence. In the other case of the node, in charging state, the charging sequence has been determined based on the present energy utilization rate instead of computation. When it finishes a unit charging period, the (1) will be reused for the computation of energy utilization rate at the present state of node charging, till the subsequent node has been identified for charging and the PCD operates with the recharging operation. It is significant to be considered that the energy utilization rate of SONRM model is timebased, denotes that the energy modification rate of any sensor node can be identified at various instances, which is the synchronized process of energy management. 


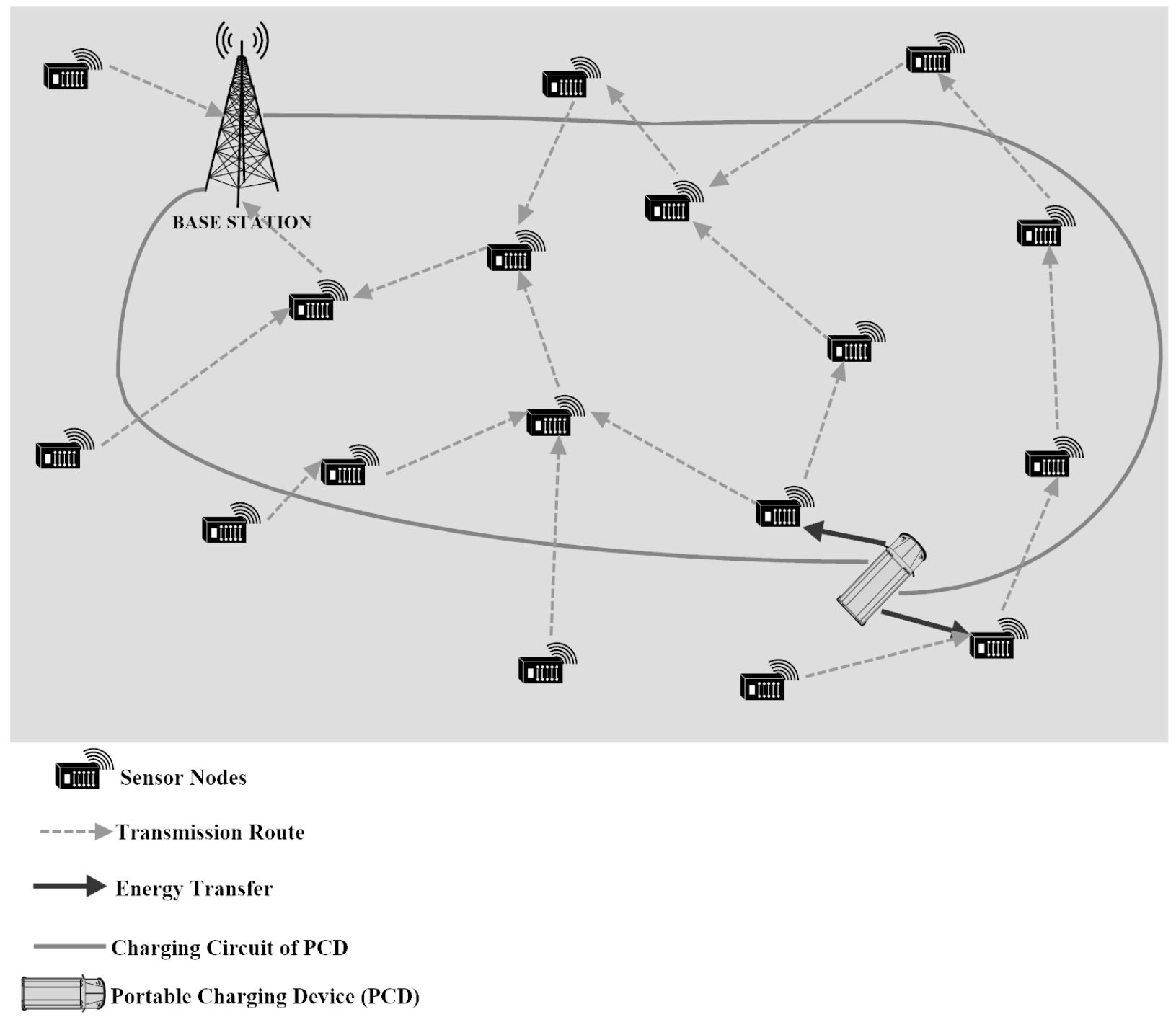

Figure. 2 System model description of the proposed work

$$
E R_{x}(t+T)=\left\{\begin{array}{c}
(1-\lambda) E R_{x}(t)+\lambda \frac{R E_{x}(t-T)-R E_{x}(t)}{T}, \text { state_of_non }- \text { charging } \\
\frac{R E_{\chi}(t-T)-R E_{x}(t)}{T}, \text { state_of_charging }
\end{array}\right.
$$

\subsubsection{Next energy starving sensor node (ESSN) selection}

Due to the reason that the PCD charges the sensor node on the basis of one-to-one charging mode, single node must be selected to recharge when receiving number of energy requests. The service schedule for corresponding request shows definite impact on all the nodes that are getting served and waiting for the service. In a case, when the present node ' $\mathrm{x}$ ' that acquires the service with low Energy utilization rate $\left(E R_{x}\right)$, which is neared to the PCD, in the interim, there is a sensor ' $y$ ' to the PCD is considerably faraway, if it is assumed as the distance between the Energy Starving Sensor Node (ESSN) to PCD. Occasionally, this can cause the dead of sensor ' $y$ ', since the energy utilization rate is too high.

In another case, when it is considered that the energy utilization rate, the present recharging node ' $\mathrm{x}$ ' with less $E R_{x}$ is farer from the PCD, in between, the node ' $y$ ' with high $E R_{y}$ than node ' $x$ ' from the buffer pool, which is nearer to the PCD, it may result in additional utilization of energy, since it is required to move front and back over the charging circuit. Hence, in the proposed model, the energy utilization rate and the distance between the current node and PCD are considered as significant parameters on selecting the next ESSN. Initially, the computational model has been designed for estimating the energy utilization rate of nodes and 
then, presented the election process of next Energy Starving Sensor Node in the WRSN.

\subsubsection{Adoption of dynamic charging threshold}

The dynamic charging threshold ' $E Y_{\text {thresh }}$ ' denotes the senor node has adequate energy to process. Further, the value $E Y_{\text {thresh }}$ lies between the maximum battery level ' $E Y_{m x}$ ' and the threshold of energy demand $E D_{\text {thresh }}$. Once the value of $E Y_{\text {thresh }}$ reaches to ' $E D_{\text {thresh }}$ ', the current recharging node stops the refilling operation. That means, it forfeits the remaining charging time to the other nodes in the network with higher rate of energy starvation, hence the node can be recharged before it suffers complete energy depletion. The value of dynamic charging threshold has been adopted based on the amount of energy requests from the buffer pool and the distributed sensors in the network. The derivation of dynamic charging is given in the Eq. (2).

$$
E Y_{\text {thresh }}=E Y_{m x}-E D_{\text {thresh }} \times \frac{\sqrt{N-x}}{\sqrt{N}}+E D_{\text {thresh }}
$$

The recharging operation stops once the node reaches the $E D_{\text {thresh }}$ and the remaining nodes with less residual energy can get the PCD service, which makes more number of nodes to be recharged per charging cycle in scheduled manner.

\subsubsection{Recharge scheduling algorithm (RSA)}

In practical, the sensor nodes having higher rate of energy utilization poses greater charging occurrence. However, the nodes nearer to the PCD stay for a shorter service period, whereas, the PCD also expends minimum energy on charging circuit motion. As mentioned earlier, both the rate of energy utilization and the distance of the PCD location have been taken into consideration for identifying the next node to be charged. Hence, the unnecessary charging delay can be effectively reduced and lifetime of the network has been prolonged.

The sensor nodes that are on energy demand are ordered based on the rate of energy utilization and the sorted results are provided is termed as ' $N_{E U}$ '. The $\mathrm{x}^{\text {th }}$ node from the set of ' $N_{E U}$ ' is mentioned as $N_{E U}(x)$. The energy utilization rate is inversely proportional to the ordered number in $N_{E U}$, which means, the node has the smaller sort number may have the chance to be as the next recharging node. The distance from the sensors in buffer pool to the PCD are ordered and provided as $N_{D i s}$, correspondingly, for node $\mathrm{x}$, it is given as $N_{D i s}(x)$.

The concrete charging schedule of the node is decided based on the weight assessment of each node, which is termed as ' $\mathrm{W}(\mathrm{x})$ '. The assessment of node weight has been calculated with the energy utilization rate and the sorting number. Hence, the weight assessment of node $\mathrm{x}$ is computed by the following Eq. (3).

$$
W(x)=\alpha N_{D i s}(x)+N_{E U}(x)
$$

Where, ' $\alpha$ ' is the control parameter, ranges from 0 to 1 to neutralize the distance effect. The value of ' $\alpha$ ' is determined on the basis of network size and node numbers. Moreover, the sensor nodes with lesser value of $\mathrm{W}(\mathrm{x})$ attain the higher charging probability. Hence, the PCD chooses the node with least W(x) value at each instant to recharge next, thereby frames the charging circuit. In some cases, multiple nodes may have equal $\mathrm{W}(\mathrm{x})$ and to handle this, nodes residual energy is compared for decision making.

At the end of the each node selection process cycle, single energy request is selected to serve and at each beginning of the cycle, the previous sequence is cleared since the energy utilization rate and distance spaces are changing frequently. The Table 1 presents the Recharge Scheduling Algorithm (RSA) for efficient operation of the proposed SONRM.

Hence, the RSA in the proposed model defines that the recharging made with real-time charging circuit results better efficiency than using pre-set path. Moreover, due to this efficient scheduling based on-demand recharging enhances the network longevity and increases the sensor nodes service time.

Table 1. Recharge scheduling algorithm for SONRM

Input: $\mathbf{N}$ (number of distributed rechargeable sensor nodes), $\mathbf{N}_{\mathbf{x}}$ (Node_ID), $\boldsymbol{E} \boldsymbol{R}_{\boldsymbol{x}}(\boldsymbol{t})$ - Energy

Utilization rate of node ' $\mathrm{x}$ ' and $\boldsymbol{N}_{\text {Dis }}$ (distance between the PCD and node 'x')

Output: next Energy Starving Sensor Node (ESSN) and PCD circuit for recharge ToSelect ESSN 


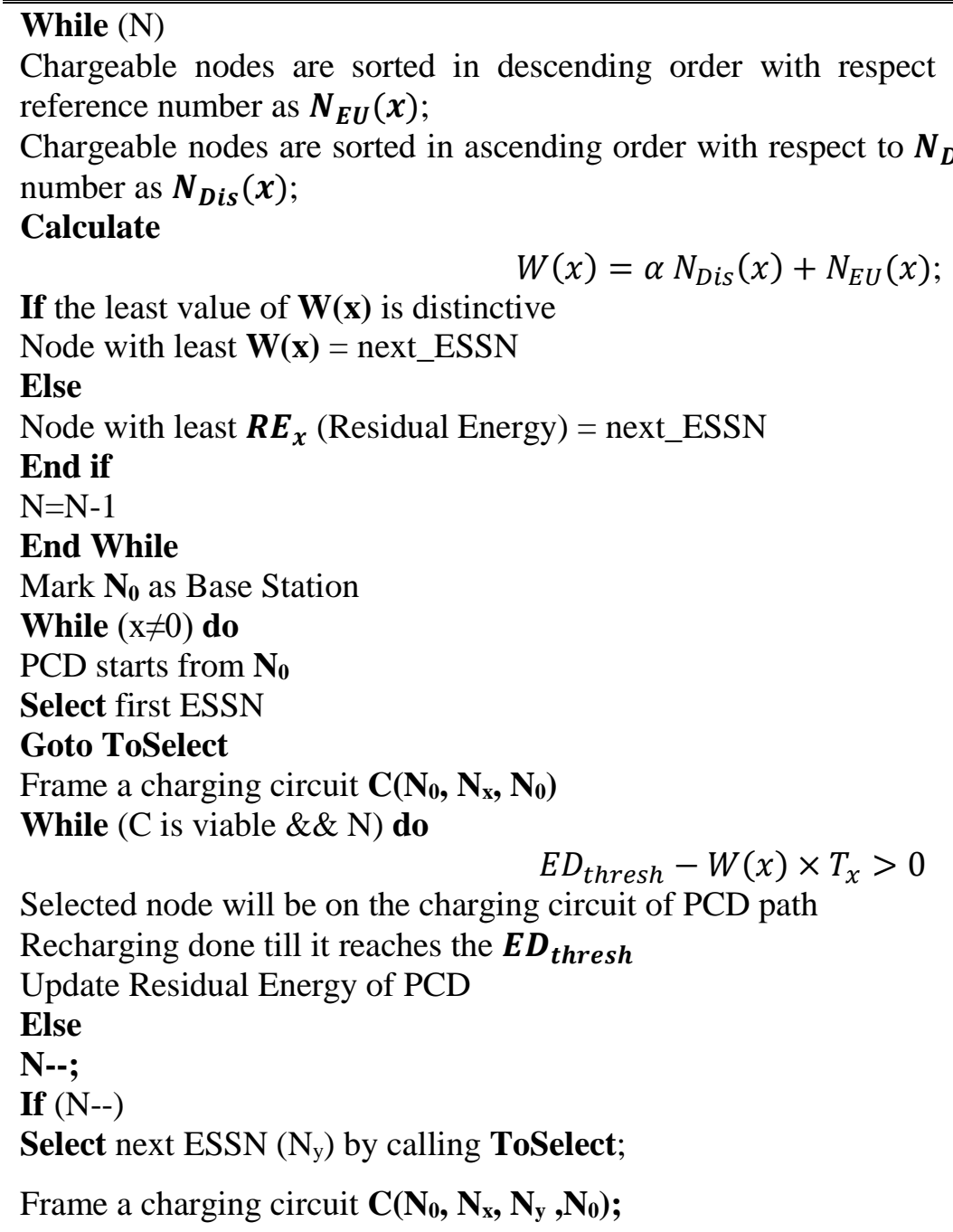

End if

End if

End While

PCD comes back to the BS $\left(\mathbf{N}_{\mathbf{0}}\right)$ for energy refilling

End While

\section{Results and discussions}

The performance of the Schedule-based Optimized Node Recharging Model (SONRM) with the incorporation of Recharge Scheduling Algorithm (RSA) has been evaluated and the obtained results are compared with the two existing approaches, Enabling Temporal and Distantial Priority (TADP) [17] and Hybrid Clustering Charging Algorithm (HCCA) [23]. Moreover, the examination has been made on the factors such as throughput, average service time, recharging time, charging efficiency and number of active nodes.
The evaluation has been accomplished with the NS-3 Network Simulation tool based on the initial parameter assumptions as given in Table 2. The simulation area taken here is $500 \times 500 \mathrm{~m}^{2}$ and every senor node is provided with the initial energy 5Joules. It is to be assumed that the data gathering process utilizes $5 \mathrm{~nJ}$ per bit. The values of the control parameters ' $\lambda$ ' and ' $\alpha$ ' are taken as 0.6 and 0.8 respectively, for neutralizing the impact of distance between nodes. That is, statistical variation among the space between sensors and energy utilization are considerably low, to set the values ranges ( 0 to 1$)$. 
Table 2. Simulation parameter with initial setup

\begin{tabular}{|l|l|}
\hline SIMULATION PARAMETERS & INITIAL SETUP \\
\hline Simulator & NS-3 \\
\hline Sensing area (Simulation area) & $500 \mathrm{~m}$ x 500m \\
\hline Simulation Time & $800 \mathrm{~s}$ \\
\hline No. of nodes & Varies from 10-100 \\
\hline Simulation End Time & 50.0 \\
\hline Mobility Model & Random Waypoint \\
\hline MAC type & IEEE 802.11 \\
\hline Traffic type & CBR \\
\hline Mobility speed of node & $5 \mathrm{~m} / \mathrm{s}$ \\
\hline Pause Time & $0 \mathrm{~s}$ \\
\hline Payload Size & $512 \mathrm{bytes}$ \\
\hline Moving Consumption & $10 \mathrm{~J} / \mathrm{m}$ \\
\hline Recharging Rate & $5 \mathrm{w} / \mathrm{s}$ \\
\hline Energy capacity of PCD & $50,000 \mathrm{~J}$ \\
\hline Mobility speed of PCD & $3 \mathrm{~m} / \mathrm{s}$ \\
\hline Energy Request Threshold & $225 \mathrm{~J}$ \\
\hline Transmission Range & $250 \mathrm{~m}$ \\
\hline Initial Energy & $5 \mathrm{Joules} \mathrm{per} \mathrm{Node}$ \\
\hline Frequency & $9 \mathrm{Mhz}$ \\
\hline
\end{tabular}

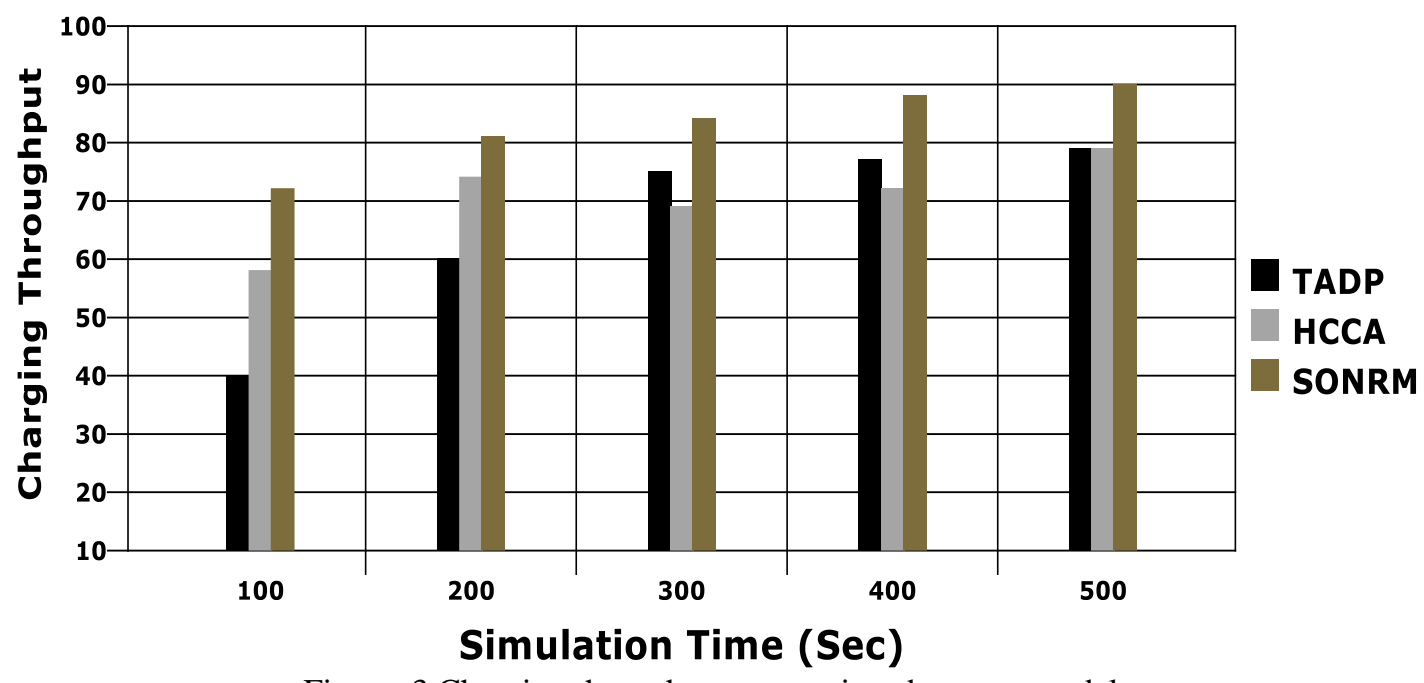

Figure. 3 Charging throughput comparison between models

The important factor for evaluating the performance of the proposed SONRM is the charging throughput, which is defined as the number of sensor nodes recharged to satisfy their energy demand at each interval of time. The Fig. 3 presents the comparison between the results of charging throughput, achieved by TADP, HCCA and SONRM. It can be stated that the simulation time increases, the value of charging thresholds also rises. Thereby, it increases the network lifetime efficiently by reducing the waiting time of nodes to get charged. 


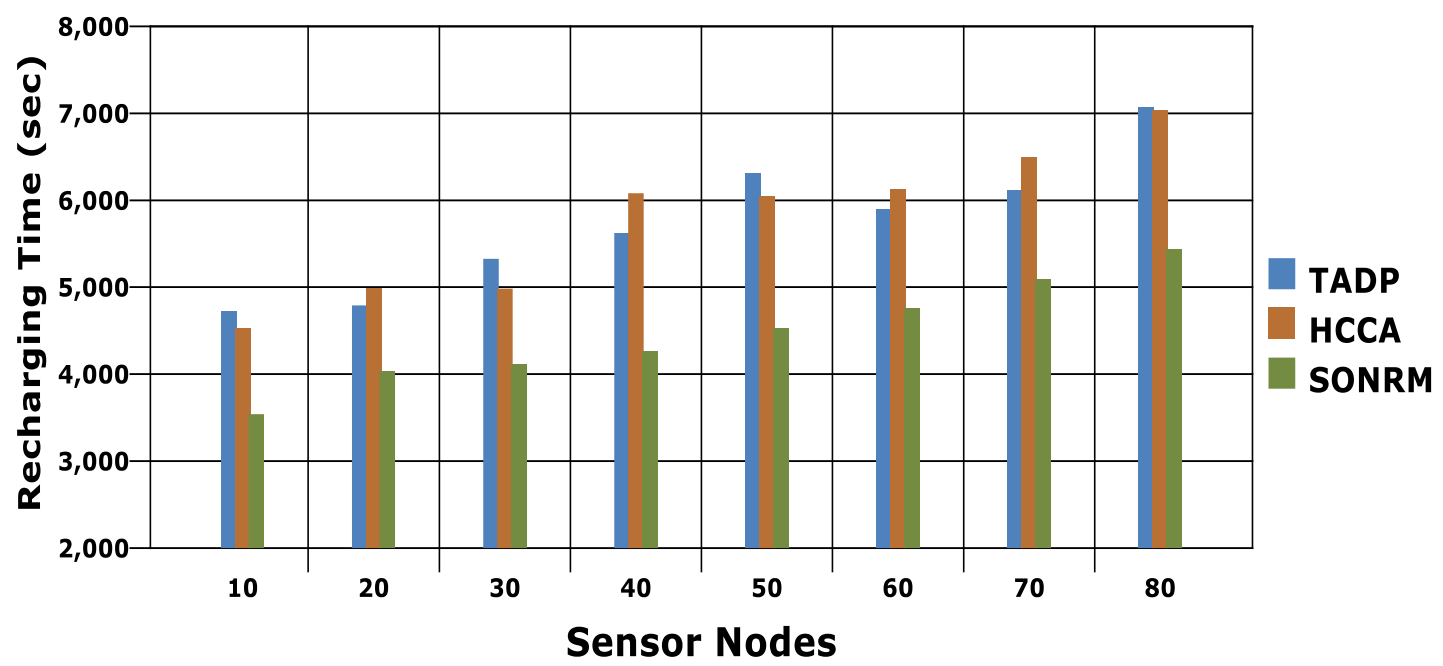

Figure. 4 Recharging time utilized by different models

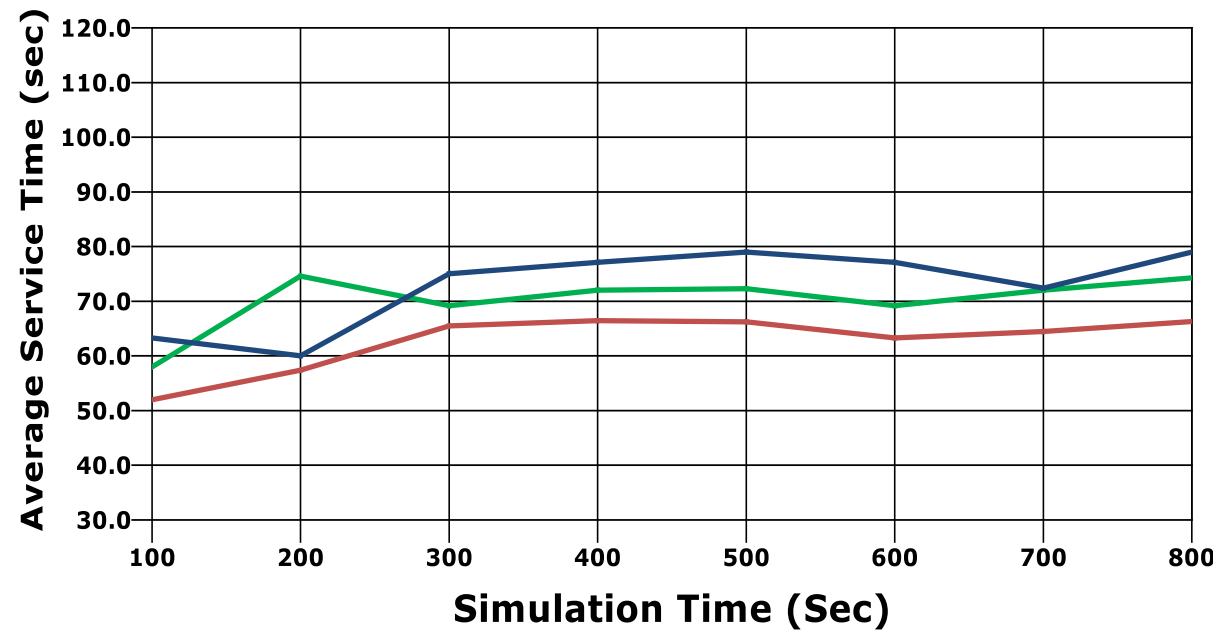

TADP

HCCA

SONRM

Figure. 5 Average service time analysis

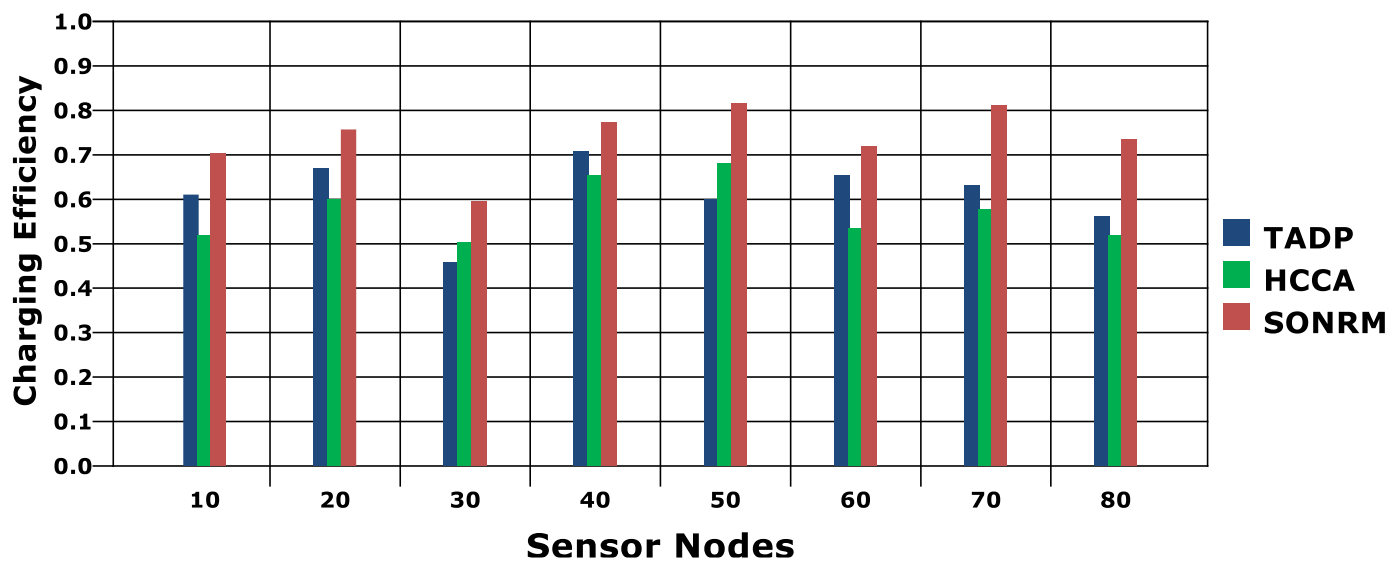

Figure. 6 Charging efficiency comparison between models

Among three models, the proposed SONRM achieves higher result of charging threshold than others.

The recharging time is another decisive parameter to evaluate the efficiency of the affirmed work. Since, the node recharging gets completed once the node attains the value of Energy demand threshold, the recharging time can be optimized in an efficient manner. The analysis of the estimated results is given in the Fig. 4. Following, the graph presented in Fig. 5 provides the comparison for average service time among models TADP, HCCA and SONRM. Here, the average service time is 


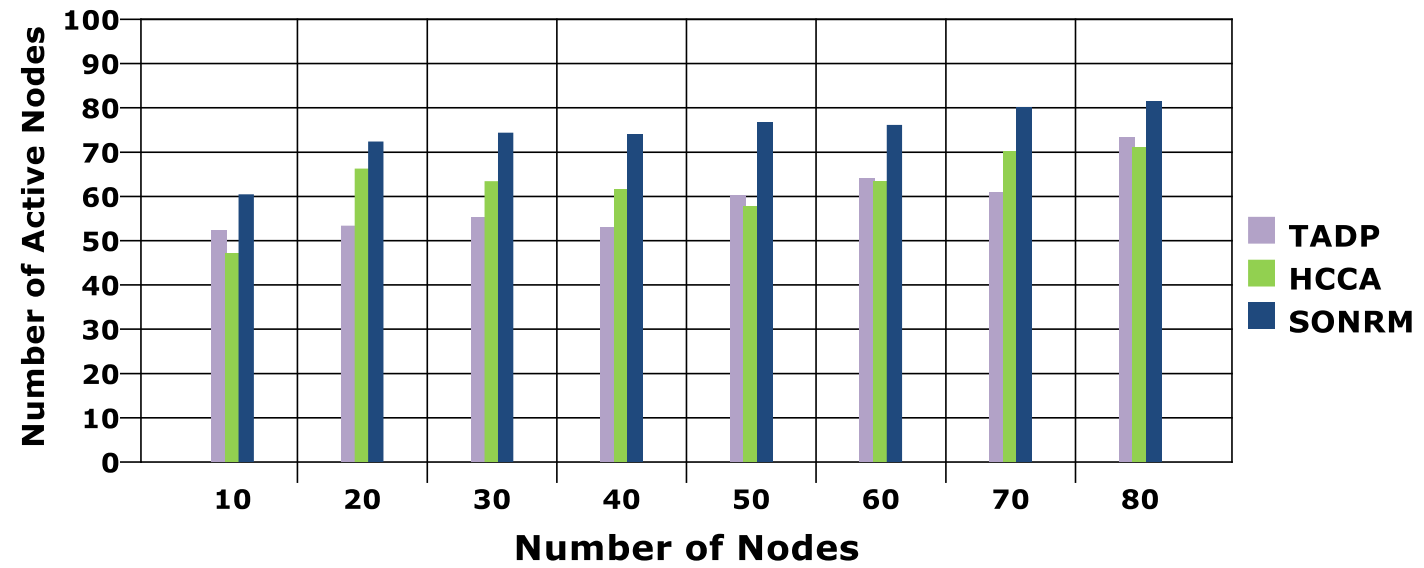

Figure. 7 Number of actual nodes vs. active nodes

defined as the time taken by the PCD to provide service to the energy starving sensor node. When the average service time is low, the waiting time of a sensor for energy service becomes minimized and the sooner energy depletion issue will be less. From the Fig. 5, it is very obvious that the proposed SONRM based recharging provides lower value of average service time among all. Therefore, it reduces the node death probability and increases the lifetime of WRSN.

The evaluation on charging efficiency with respect to various number of sensor nodes is provided in the Fig. 6. It is clearly noted from the evaluation results that the recharging process of the proposed model is more proficient than the other two. In average, SONRM provides $36 \%$ of higher charging efficiency than others. This is achieved with the efficient framing of Recharge Scheduling Algorithm, which considers energy utilization rate, PCD capacity of recharging and appropriate selection of next ESSN to mark the charging circuit.

The network longevity is the most significant factor to be focussed on developing a model for WRSN. This can be evaluated by analysing the number of active nodes presented in the defined WRSN when the simulation goes on. The results are illustrated in the Fig. 7. From the results, it is shown that the SONRM model makes more number of nodes to be active, thereby, reduces the node death due to the energy exhaustion and increases the network lifespan.

\section{Conclusion and future work}

This paper provides a novel method for efficient on-demand recharging of distributed sensor nodes in WRSN. In the proposed Schedule-based Optimized Node Recharging Model (SONRM), a proficient Recharge Scheduling Algorithm (RSA) for selecting appropriate Energy Starving Sensor Node (ESSN) has been framed to make the feasible charging circuit for Portable Charging Device (PCD) to travel. The PCD recharges the sensor nodes, when there is an energy demand, before their complete energy depletion occurs, to sustain the network longevity. This can be effectively accomplished with the incorporation of RSA. Though the path selection process for PCD is very complicated since the energy utilization rate of nodes and the PCD location varies with respect to time, adoption of Dynamic Charging Threshold makes it to handle in effective manner. The simulation results have shown the proposed SONRM provides better performance than the compared TADP and ECCA models, based on the factors such as charging throughput, recharging time, efficiency, throughput and number of active nodes in the defined network. The overall charging efficiency has been enhanced for about $36 \%$ and the number of active nodes in the proposed model is comparatively more than other, which increases the network lifetime efficiently.

As future work, this model can be further enhanced by employing multiple PCDs for recharging the nodes with energy demands in largescale networks.

\section{References}

[1] S. Soro and W. B. Heinzelman, "Cluster head election techniques for coverage preservation in wireless sensor networks," Ad Hoc Networks, Vol. 7, No. 5, pp. 955-972, 2009.

[2] C. Wang, J. Li, Y. Yang, and F. Ye, "Combining solar energy harvesting with wireless charging for hybrid wireless sensor networks", IEEE Transactions on Mobile Computing, Vol. 17, No. 3, pp. 560-576, 2017.

[3] G. Anastasi, M. Conti, M. Di Francesco, and A. Passarella, "Energy conservation in wireless 
sensor networks: a survey", Ad Hoc Networks, Vol. 7, No. 3, pp. 537-568, 2009.

[4] I. F. Akyildiz, W. Su, Y. Sankarasubramaniam, and E. Cayirci, "Wireless sensor networks: a survey", Computer Networks, Vol. 38, No. 4, pp. 393-422, 2002.

[5] W. K. G. Seah, Z. A. Eu, and H.-P. Tan, "Wireless sensor networks powered by ambient energy harvesting (WSN-HEAP) - survey and challenges", In: Proc. of the 1st International Conference on Wireless Communication, Vehicular Technology, Information Theory and Aerospace \& Electronics Systems Technology, pp. 1-5, 2009.

[6] L. J. Chien, M. Drieberg, P. Sebastian, and L. H. Hiung, "A simple solar energy harvester for wireless sensor networks", In: Proc. of the 6th International Conference on Intelligent and Advanced Systems, pp. 1-6, 2016.

[7] I. F. Akyildiz, W. Su, Y. Sankarasubramaniam, and E. Cayirci, "A survey on sensor networks", IEEE Communications Magazine, Vol. 40, No. 8, pp. 102-105, 2002.

[8] J. Li, M. Zhao, and Y. Yang, "A novel energy replenishment and data gathering mechanism in wireless rechargeable sensor networks", In: Proc. of the IEEE Global Communications Conference, pp. 5350-5355, 2012.

[9] P. Zhong, Y. Li, W. Liu, G. Duan, Y. Chen, and N. Xiong, "Joint mobile data collection and wireless energy transfer in wireless rechargeable sensor networks", Sensors, Vol. 17, pp. 1881, 2017.

[10] A. Liu, M. Huang, M. Zhao, and T. Wang, "A smart high-speed backbone path construction approach for energy and delay optimization in WSNs", IEEE Access, Vol. 6, pp. 13836-13854, 2018.

[11] J. Gao, J. Wang, P. Zhong, and H. Wang, “On Threshold free Error Detection for Industrial Wireless Sensor Networks", IEEE Trans. Ind. Inf., Vol. 14, pp. 2199-2209, 2018.

[12] J. Wang, T. Si, X. Wu, X. Hu, and Y. Yang, "Sustaining a perpetual wireless sensor network by multiple on-demand mobile wireless chargers", In: Proc. of the IEEE International Conference on Networking, Sensing and Control, pp. 533-538, 2015

[13] C. Lin, Y. Wu, Z. Liu, M. Obaidat, C. Yu, and $\mathrm{G}$. $\mathrm{Wu}$, "A game theoretical collaborative charging scheme for wireless rechargeable sensor networks", J. Syst. Softw., Vol. 121, pp. 88-104, 2016.

[14] Y. Peng, Z. Li, W. Zhang, and D. Qiao, "Prolonging sensor network lifetime through wireless Charging", In: Proc. of the IEEE RealTime Systems Symposium, pp. 129-139, 2010.

[15] L. He, Y. Gu, J. Pan, and T. Zhu, "On-demand charging in wireless sensor networks: Theories and applications", In: Proc. of the Mobile Ad Hoc and Sensor System, pp. 28-36, 2013.

[16] L. He, L. Kong, Y. Gu, J. Pan, and T. Zhu, "Evaluating the on-demand mobile charging in wireless sensor networks", IEEE Trans. Mob. Comput., Vol. 9, pp. 1861-1875, 2015.

[17] C. Lin, Z. Wang, D. Han, Y. Wu, C. Yu, and G. $\mathrm{Wu}$, "TADP: Enabling temporal and distantial priority scheduling for on-demand charging architecture in wireless rechargeable sensor networks", J. Syst. Archit., Vol. 70, pp. 26-38, 2016.

[18] C. Lin, D. Han, J. Deng, and G. Wu, "P2S: A primary and passer-by scheduling algorithm for on-demand charging architecture in wireless rechargeable sensor networks", IEEE Trans. Veh. Technol., Vol. 66, pp. 8047-8058, 2017.

[19] G. Jiang, S. Lam, Y. Sun, L. Tu, and J. Wu, "Joint charging tour planning and depot positioning for wireless sensor networks using mobile chargers", IEEE/ACM Trans. Netw., 25, 2250-2266, 2017.

[20] C. Hu and Y. Wang, "Schedulability decision of charging missions in wireless rechargeable sensor networks", In: Proc. of the IEEE International Conference on Sensing, Communication Network, pp. 450-458, 2014.

[21]X. Ye and W. Liang, "Charging utility maximization in wireless rechargeable sensor networks", Wirel. Netw., Vol. 23, pp.20692081, 2016.

[22] X. Rao, P. Yang, Y. Yan, H. Zhou, and X. Wu, "Optimal recharging with practical considerations in wireless rechargeable sensor network", IEEE Access, Vol. 5, pp. 4401-4409, 2017.

[23] C. Lin, G. W. Wu, M. S. Obaidat, and C. W. $\mathrm{Yu}$, "Clustering and splitting charging algorithms for large scaled wireless rechargeable sensor networks", J. Syst. Softw., Vol. 113, pp. 381-394, 2016. 